\title{
Diagnostik und Therapie von ZNS-Infektionen bei pädiatrischen onkologischen Patienten
}

\author{
P. Hernárz Driever \\ S. Reich
}

\author{
Diagnosis and Management of Central Nervous System Infections \\ in Pediatric Cancer Patients
}

\section{Zusammenfassung}

Infektionen des zentralen Nervensystems (ZNS) sind eine der häufigsten Ursachen für neurologische Defizite bei pädiatrisch-onkologischen Patienten. Nicht immer manifestieren sich die Symptome eindeutig und erlauben daher keine scharfe Trennung von mannigfaltigen Differenzialdiagnosen. Die wiederholte klinischneurologische Untersuchung ermöglicht die gezielte Auswahl der notwendigen laborchemischen und neuroradiologischen Maßnahmen sowie der Erregerdiagnostik. Aufgrund der hohen Mortalität sowie Folgemorbidität bei verzögert antiinfektiös behandelten Patienten ist eine empirische Therapie mit adäquaten Dosen notwendig, bis diagnostische Hinweise zur spezifischen Behandlung führen. Die vorliegende Arbeit enthält Empfehlungen der Arbeitsgruppe „Infektionen bei immunsupprimierten Kindern“ der Deutschen Gesellschaft für Pädiatrische Infektiologie (DGPI) und der Deutschen Gesellschaft für Pädiatrische Onkologie und Hämatologie (GPOH) zu Diagnosik und Therapie von ZNS-Infektionen bei immunsupprimierten Kindern.

\section{Schlüsselwörter}

Zentrales Nervensystem · Infektionen · Onkologie · Kinder

\section{Abstract}

Infections of the central nervous system (CNS) are a common cause of neurological abnormalities in pediatric oncology patients apart from cancer disease of the CNS. Often symptoms do not present unequivocally, impeding a clear distinction from other differential diagnosis. Repeated neurological examinations of the patient allow selecting the necessary laboratory tests and imaging techniques. As delayed antiinfectious treatment is associated with high mortality and severe sequelae of survivors, patients do need empirical therapy using adequate doses until diagnostic results allow a more specific treatment. This article provides the recommendations of the Infectious Diseases Working Party of the German Society for Pediatric Infectious Diseases (DGPI) and the German Society for Pediatric Hematology/Oncology ( $\mathrm{GPOH})$ for diagnosis and treatment of infections of the CNS in immunocompromised patients.

\section{Key words}

Central nervous system $\cdot$ infections $\cdot$ oncology $\cdot$ children

\section{Einleitung}

Die Bedeutung neurologischer Affektionen bei pädiatrisch-onkologischen Patienten im Rahmen der Erkrankung und ihrer Behandlung nimmt infolge der verbesserten Überlebenschancen bezüglich der Grunderkrankung zu. Die häufigste Differenzial- diagnose neurologischer Defizite sind die tumorassoziierten Affektionen des ZNS, d.h. Metastasen und primäre ZNS-Tumoren. Infektionen des ZNS sind eine sehr häufige und wichtige nicht onkologische Ursache von ZNS-Erkrankungen bei pädiatrischonkologischen Patienten. Die klinischen Symptome immunkompetenter Patienten können bei immunsupprimierten Patienten

Institutsangaben

Klinik für Pädiatrie mit Schwerpunkt Onkologie und Hämatologie, Charité-Universitätsmedizin Berlin

Korrespondenzadresse

Dr. Pablo Hernáiz Driever · Klinik für Pädiatrie mit Schwerpunkt Onkologie und Hämatologie .

Charité-Universitätsmedizin Berlin · Augustenburger Platz 1 · D-13353 Berlin · Tel.: 030/4 50666173 .

Fax: 030/4 50566906·E-mail: pablo.hernaiz@charite.de

Bibliografie

Klin Pädiatr 2005; 217 (Suppl 1): S120-S129 • (c) Georg Thieme Verlag KG Stuttgart · New York

DOI $10.1055 / \mathrm{s}-2005-872506$

ISSN 0300-8630 
sich nur leicht bemerkbar machen oder ganz fehlen. Außerdem können eine Kortikosteroidtherapie und nicht-steroidale antiinflammatorische Substanzen das wichtige Symptom Fieber unterdrücken. Die Patienten bedürfen daher einer wiederholten neurologischen Untersuchung, um frühzeitig sinnvolle laborchemische Bestimmungen, adäquate bildgebende Verfahren, spezielle Erregernachweise und folgerichtige neurophysiologische Diagnostik durchzuführen. Die klinische und laborchemische Konstellation, die bildgebenden Verfahren und der Verlauf bestimmen, ob es sich um eine Meningitis, Enzephalitis, Hirnabszess oder eine Shuntinfektion handelt. Die häufigste Infektionsform ist unverändert die Meningitis [53]. In der Regel ist bei neurologischen Auffälligkeiten primär eine infektiöse Genese differenzialdiagnostisch kaum auszuschließen und muss daher umgehend empirisch behandelt werden. Dies gilt insbesondere für Patienten in der Granulozytopenie, die eine signifikant höhere Mortalität und Morbidität im Vergleich zu nicht granulozytopenen onkologischen Patienten aufwiesen [62]. Die Erarbeitung von Empfehlungen ist erschwert, da für das pädiatrisch onkologische Patientengut eine minimale kontrollierte Datenmenge existiert, Kasuistiken dominieren, und zudem die vorhandenen Informationen entweder von Erwachsenen oder der Behandlung von nicht onkologischen immunkompetenten Kindern und Jugendlichen stammen.

\section{Definition}

Infolge der Infektion gehen bei Viren aufgrund des Tropismus spezifisch und bei anderen Erregern unspezifisch Glia-, Ganglienzellen und/oder Gefäßbindegewebszellen zu Grunde und es kommt zur Exsudation aus den Gefäßen sowie zur glialen Proliferation. Die Einteilung der Krankheitsform erfolgt nach Erreger und Lokalisation. Aufgrund der mangelnden anatomischen Abgrenzung sind die Übergänge fließend. Bei der Meningitis liegt das entzündliche Exsudat im Subarachnoidalraum. Entlang der pialen Gefäße kann die Entzündung auf die Hirnrinde übergreifen und wird so zur Meningoenzephalitis. Die seröse Leptomeningitis weist fast ausschließlich eine Hyperämie mit vermehrter Liquorproduktion auf. Im Gegensatz dazu sind Enzephalitiden Entzündungen, die auf das Hirngewebe bezogen sind und außer per continuitatem von den Meningen auch hämatogenen Ursprungs sein können. Herrscht ein Markscheidenzerfall vor, spricht man von einer Leukoenzephalitis. Im Gegensatz dazu ist eine die graue Substanz befallende Entzündung eine Polioenzephalitis. Einen Befall der Substantia grisea et alba nennt man Panenzephalitis. Bei einer Myelitis ist vorwiegend das Rückenmark befallen. Als Abszess werden umschriebene Infektionen des Gehirngewebes bezeichnet. Im Rahmen einer Shuntinfektion gilt der Fremdkörper als Ausgangspunkt für eine ZNS-Infektion, die von einer Ventrikulitis über Meningitis bis zum Abszess oder Meningoenzephalitis reichen kann.

\section{Epidemiologie, Risikofaktoren}

Als primäre Risikofaktoren für eine Infektion des ZNS im pädiatrisch onkologischen Patientengut gelten:

- Antitumorale Therapie [62]

- Neurochirurgische Eingriffe [62]
Tab. 1 Wichtigste Differenzialdiagnosen bei neurologischen Auffälligkeiten pädiatrisch onkologischer Patienten
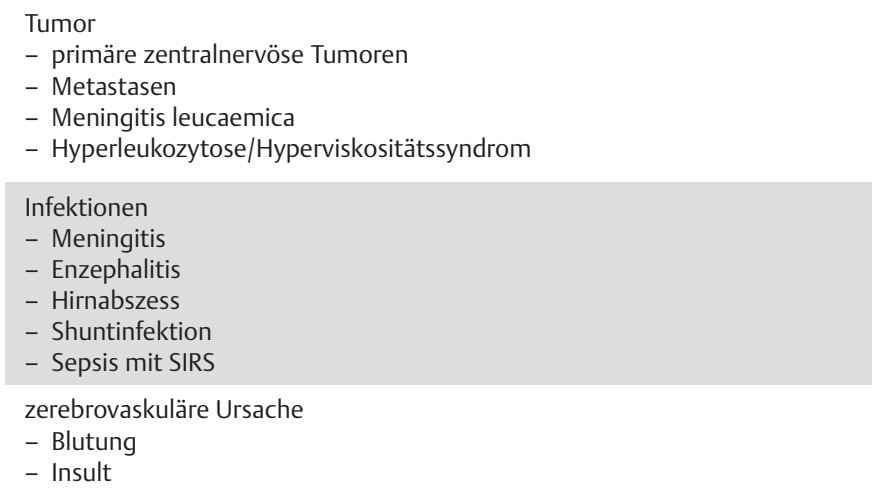

Krampfanfall/postiktaler Schlaf

behandlungsassoziiert

- Zytostatika

- Methotrexat

- Ara-C

- Ifosfamid

- 5-Fluorouracil

- Cyclosporin A

- Narkotika

- Benzodiazepine

- Antihistaminika

- Antiepileptika

- trizyklische Antidepressiva

- Leukenzephalopathie

metabolische Veränderungen

- Hyponatriämie (SIADH)

- Hypoglykämie/Hyperglykämie

- Hypomagnesiämie

- Urämie

- Leberversagen

Bestrahlungsreaktion

kardiovaskuläre Ursachen

- Hypotension/Bluthochdruck

- Dehydratation

- Hypoxie

- Anämie

Depression

- Intraventrikuläre Behandlung mit Chemotherapie: Infektionen von intrazerebralen Kathetern manifestieren sich mit einer Prävalenz von 2-23\% der Patienten [12, 41, 62]. Die Liste der häufigsten Erreger ist in Tab. 4 dargestellt [41]. Die Häufigkeit der Nutzung der intrazerebralen Katheter korreliert nicht mit der Inzidenz der Shuntinfektionen [41, 51].

- Alter: In einer prospektiven Untersuchung zu Fungämien bei onkologischen Patienten waren pädiatrische Patienten im Vergleich zu erwachsenen Patienten mit einer zehnfach höheren Inzidenz der Entwicklung einer meningitischen Manifestation (11,4 vs. 0,8\%) belastet [30]. Bezüglich des Erregerspektrums waren in einer Serie grampositive Erreger bei pädiatrisch onkologischen Patienten dominierend sowie selten Listerien nachweisbar, während bei Erwachsenen gramnegative Erreger und Listerien vorherrschten [62]. Bei immunkompetenten Patienten haben pädiatrische Patienten mit bakterieller Meningitis im Vergleich zu Erwachsenen eine deutlich niedrigere Mortalität und Morbidität [2]. 
Tab. 2 Erweitertes diagnostisches Vorgehen bei neurologischen Auffälligkeiten (siehe auch Kapitel FUO)

\begin{tabular}{ll}
\hline Analyse & Fragestellung \\
\hline Blutzucker & Hyper- oder Hypoglykämie \\
\hline Blutbild & Anämie, Hyperleukozytose, absolute Neutropenie \\
\hline Kreatinin & Nierenfunktionsstörung \\
\hline ALAT/ASAT, Bilirubin, & Leberfunktionsstörung \\
\hline NH3 & Herzmuskelschädigung, zerebrale Ischämie \\
\hline CK & Exsikkose, Elektrolytstörung \\
\hline $\begin{array}{l}\text { Na, K, Ca, Mg, P, } \\
\text { Harnstoff }\end{array}$ & $\begin{array}{l}\text { Zellzahl, Zellmorphologie, Eiweißvermehrung, } \\
\text { Einblutung, Zucker- und Laktatveränderung }\end{array}$ \\
\hline Liquor & respiratorische Störung, metabolische Störung \\
\hline Blutgase & $\begin{array}{l}\text { Leberfunktionsstörung, Verbrauchskoagulopathie } \\
\text { bei disseminierter intravasaler Gerinnungsstörung }\end{array}$ \\
\hline Gerinnung & iatrogene Schädigung durch Überdosierung \\
\hline (Chemotherapie und andere Medikamente)
\end{tabular}

\section{Neurologische Symptomatik}

Die neurologischen Symptome können allgemein-neurologisch bis hin zur schweren Enzephalopathie und/oder mit fokalen Ausfällen imponieren. Bei bewusstseinsgetrübten Patienten muss zuerst die Komatiefe eingeschätzt werden, Paresen aufgedeckt, die Nackensteifigkeit geprüft und eine Fundoskopie durchgeführt werden. Weite, Form und Lichtreaktion der Pupillen geben wichtige Informationen über den Reflexkreis des II. und III. Hirnnerven. Tab. 3 gibt eine Übersicht zur Symptomenkonstellation der einzelnen Entzündungsformen. Diese Krankheitsabfolge setzt allerdings ein immunkompetentes System voraus und kommt bei onkologischen Patienten eher selten vor [62]. Bei diesen sind die Symptome in der Regel mitigiert, da die für die Symptome notwendigen Entzündungsreaktionen häufig fehlen. Zusätzlich zur neurologischen Untersuchung ist eine HNO- und augenärztliche Untersuchung zur Fokussuche und Funktionsprüfung unabdingbar. Im Weiteren erfolgt die sonst übliche Fokussuche klinisch und mittels zusätzlicher Untersuchungen (Rö-Thorax, Sono-Abdomen, Echokardiographie). Hierbei ist zu bedenken, dass in einer Serie $40 \%$ der Patienten mit einer ZNS-Infektion extraneurale Infektionen mit einem 2. Keim aufwiesen [53].

\section{Laborchemische Diagnose im Blut und Liquor}

Die mikrobiologische Diagnostik umfasst immer die Anlage von Kulturen aus Liquor cerebrospinalis sowie Blut. Es sollte immer der Versuch eines Direktnachweises mittels Anfärbung von Bakterien und Pilzen erfolgen. Ein routinemäßiger Antigennachweis durch kommerziell verfügbarer Latexagglutinationsteste bei mikroskopisch unauffälligem Liquor ist nicht sinnvoll. Der Wert von Viruskulturen aus Liquor, Stuhl oder Rachenspülwasser ist anbetrachts der wachsenden Möglichkeiten der PCR-Methoden zum Direktnachweis des Erregergenoms rückläufig. Für virologische und molekularbiologische Untersuchungen sollten größere Volumina gesammelt werden. Bei jedem Verdacht sollten auch Serumproben gewonnen werden, um infektionsserologische Untersuchungen im Verlauf zu ermöglichen [42].

Die laborchemische Diagnostik des Liquors umfasst Zellzahl, Differenzierung, Laktat, Liquor-Serum-Glukosequotient, Gesamtprotein im Liquor und in Serum. Eine Elektrophorese von Serum und Liquor ermöglicht die Diagnose einer Schrankenstörung und die Abgrenzung einer autochthonen Immunglobulinproduktion im Liquor. Eine Antikörperbestimmung im Liquor empfiehlt sich erst, wenn im Serum virale Antikörper vorhanden sind [42].

Bestimmungen von Akutphaseproteinen im Liquor sind routinemäßig nicht empfohlen. Für die Diagnose einer eitrigen Meningitis bei immunkompetenten Patienten ist eine granulozytäre

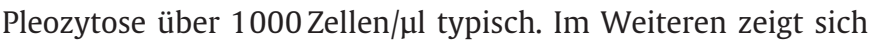
eine schwere Blut-Liquor-Schrankenstörung und eine Erniedrigung des Glukosewertes im Liquor mit meist weniger als $30 \mathrm{mg} / \mathrm{dl}$ (Liquor-Serum-Glukosequotient kleiner 0,3). Ebenso ist das Laktat im Liquor meist über 3,5 mmol/l erhöht [42]. Die Zellzahlerhöhung wie auch die Schrankenstörung ist im pädiatrisch-onkologischen Patientengut infolge einer Leuko- und Granulozytopenie sowie Kortikosteroidtherapie eher vermindert [62]. Umgekehrt werden erhöhte Zellzahlen und Proteinvermehrung häufig infolge einer chemischen Meningitis nach Knochenmarktransplantation beobachtet [70]. Im Serum sollten allgemeine Infektionsmarker bestimmt werden [47].

Tab. 3 Klinische Symptome der entzündlichen Veränderungen bei immunkompetenten Kindern

\begin{tabular}{|c|c|c|c|}
\hline Meningitis & Enzephalitis & Abszess & Shuntinfektion \\
\hline
\end{tabular}


Tab. 4 Häufigste Erreger bei klinischen Erscheinungsbildern [12, 41, 53]

\begin{tabular}{|c|c|c|c|}
\hline Meningitis & Enzephalitis & Abszess & Shuntinfektion \\
\hline $\begin{array}{l}\text { Bakterien } \\
\text { - B-Streptokokken } \\
\text { - Pneumokokken } \\
\text { - Streptococcus pyogenes } \\
\text { - treptococcus viridans } \\
\text { - Meningokokken } \\
\text { - Hämophilus influenzae } \\
\text { - E. coli } \\
\text { - Klebsiella pneumoniae } \\
\text { - Enterobacteriaceae } \\
\text { - Citrobacter }\end{array}$ & $\begin{array}{l}\text { Bakterien } \\
\text { - Listeria monocytogenes } \\
\text { - Streptokokken } \\
\text { - Mykoplasmen } \\
\text { - Gramnegative Keime }\end{array}$ & $\begin{array}{l}\text { Bakterien } \\
\text { - Staphylococcus aureus } \\
\text { - anaerobe Keime } \\
\text { - Listeria monocytogenes } \\
\text { - Nocardia asteroides }\end{array}$ & $\begin{array}{l}\text { Bakterien } \\
\text { - Staphylococcus aureus } \\
\text { - Staphylococcus epi. } \\
\text { - Alpha- und Beta-hämolysierende } \\
\text { Streptokokken } \\
\text { - Pseudomonas aeruginosa } \\
\text { - Bacillus cereus } \\
\text { - Propionibacterium acnes } \\
\text { - E. coli } \\
\text { - Klebsiella pneumoniae } \\
\text { - Corynebacterium spp. }\end{array}$ \\
\hline $\begin{array}{l}\text { Pilze } \\
\text { - C. neoformans } \\
\text { - Candida spp. }\end{array}$ & $\begin{array}{l}\text { Pilze } \\
\text { - C. neoformans } \\
\text { - Candida spp. }\end{array}$ & $\begin{array}{l}\text { Pilze } \\
\text { - Aspergillus spp. } \\
\text { - C. neoformans } \\
\text { - Mucoraceae }\end{array}$ & $\begin{array}{l}\text { Pilze } \\
\text { - Aspergillus spp. } \\
\text { - Candida spp. }\end{array}$ \\
\hline \multirow[t]{2}{*}{$\begin{array}{l}\text { Viren } \\
\text { - HSV } \\
\text { - HHV-6 } \\
\text { - CMV } \\
\text { - VZV }\end{array}$} & $\begin{array}{l}\text { Viren } \\
\text { - HSV } \\
\text { - HHV-6 } \\
\text { - CMV } \\
\text { - VZV }\end{array}$ & $\begin{array}{l}\text { Viren } \\
\text { - VZV } \\
\text { - CMV }\end{array}$ & \\
\hline & $\begin{array}{l}\text { Parasiten } \\
\text { - Toxoplasma gondii } \\
\text { - Strongyloides stercoralis }\end{array}$ & $\begin{array}{l}\text { Parasiten } \\
\text { - Toxoplasma gondii } \\
\text { - Strongyloides stercoralis }\end{array}$ & \\
\hline
\end{tabular}

Die Diagnose einer Infektion des Shuntsystems im Rahmen einer intraventrikulären Therapie ist dadurch erschwert, dass es im Liquor häufig zu einer neutrophilen Pleozytose kommt, die aber nicht spezifisch sein muss, da es sich oft um eine sterile chemische Meningitis im Rahmen der intraventrikulären antitumoralen Therapie handelt [12]. Ein Anstieg der neutrophilen Pleozytose ist jedoch hinweisend auf eine Infektion, noch bevor die aus dem Shuntsystem gewonnene Liquorkultur sich als positiv erweist [51]. Im Serum bestimmte Werte für Akutphase-Proteine bieten keine diagnostische Hilfe bei der Identifikation einer Shuntinfektion [51].

Die Biopsie von Hirn- oder meningealem Gewebe empfiehlt sich zum Ausschluss eines Tumors bei unklarer Klinik und nicht eindeutigen neuroradiologischen Untersuchungsergebnissen sowie zur Spezifizierung der Therapie. Die Indikationen liegen vor bei $[48,53]$ :

- Nachweis unspezifischer, ringförmig kontrastmittelaufnehmender Läsionen, ohne infektiologische oder onkologische Klärung mittels Liquoruntersuchung und bildgebender Verfahren

- Mangelndem Ansprechen einer als Toxoplasma-bedingt eingeordneten Läsion nach 2 bis 3 Wochen adäquater Therapie

- Progressiver Veränderung einer Auffälligkeit im ZNS, die nicht anderweitig geklärt werden konnte

- Hinweisen auf eine Vaskulitis mittels bildgebender Verfahren, die sich mittels Liquorbefunden bestätigen lässt

\section{Neuroradiologische Diagnostik}

Bildgebende Verfahren werden zum Nachweis der entzündlichen Veränderungen des Parenchyms und zur Evaluation der Folgen benötigt. Die neuroradiologischen Verfahren sollen insbesondere ein Hirnödem, einen Hydrozephalus, eine Blutung, ei- nen Hirninfarkt, eine Zerebritis, eine Ventrikulitis, einen Hirnabszess, ein subdurales Empyem, parameningeale Infektionsherde (Sinusitis, Mastoiditis), intrakranielle Luft bei Durafistel, meningeale und ventrikuläre ependymale Beteiligung sowie eine Sinusvenenthrombose und Kalzifikationen als Folge der Infektion abgrenzen. Bei offener Fontanelle bietet die Sonographie des Schädels die Möglichkeit, häufige Untersuchungen ohne Sedation durchzuführen. Einschränkungen der Interpretation sind beim Nachweis von extraaxialen Flüssigkeitsansammlungen vorhanden. Die Dopplersonographie erlaubt zusätzlich eine Beurteilung der zerebralen Durchblutung. In der Regel ist die Kernspintomographie (MRT) der Computertomographie (CT) überlegen, bei Blutungen als gleichwertig zu betrachten. Die CT hat ihre Stärke in der Beurteilung von Kalzifikationen, z.B. infolge Toxoplasmose, und knöcherner Strukturen, sowie der Schnelligkeit bei der Abgrenzung von Flüssigkeitsansammlungen. Die MRT erlaubt jedoch eine bessere Unterscheidung von weißer und grauer Substanz, eine genauere Abgrenzung im Bereich der hinteren Schädelgrube, eine verbesserte Auflösung bei leptomeningealer Erkrankung und eine exaktere Diagnostik einer Sinusvenenthrombose. Umgekehrt hat die CT in akuten Situationen aufgrund ihrer Schnelligkeit Vorteile gegenüber der MRT. Da entzündliche Komponenten beurteilt werden müssen, ist immer die Kontrastmittelgabe obligat. Wenn möglich sollte neben der $\mathrm{T}_{1}$-Wichtung eine $\mathrm{T}_{2}$-Wichtung sowie eine diffusionsgewichtete MRT durchgeführt werden. Sinnvoll ist, wenn möglich, eine MRAngiographie anfertigen zu lassen. CT als auch MRT kommen bei pädiatrisch-onkologischen Patienten an die Grenzen bezüglich ihrer Aussagekraft, da Kortikosteroide die Kontrastmittelaufnahme vermindern und erniedrigte Hämoglobinwerte den Nachweis und die Beurteilung des Alters intrakranieller Blutungen einschränken. Neuere Methoden wie MR-Spektroskopie und PET ermöglichen zum Teil die verbesserte Abgrenzung von Tumor, Infektion und Strahlenfolge [2, 25, 53]. 


\section{Neurophysiologische Diagnostik}

Ein EEG ist zum Nachweis einer fokalen oder diffusen Hirnfunktionsstörung vor allem bei klinisch noch unklarer Situation erforderlich. Es ermöglicht die Abgrenzung zu einer medikamentenbedingten Funktionsstörung, den Ausschluss eines nichtkonvulsiven Status epilepticus und den Ausschluss einer dissoziativen Bewusstseinsstörung (dann normales EEG). Bei komatösen Patienten ermöglichen somatosensibel evozierte Potenziale Aussagen zur Funktionsfähigkeit des motorischen Systems und akustisch evozierte Potenziale Aussagen zur Funktion des Hirnstamms. Zusätzlich können akustisch evozierte Potenziale zusammen mit kalorischer Prüfung Hinweise auf die Störung des vestibulokochleären Systems geben.

\section{Antibiotische Therapie}

\section{Pharmakokinetische Aspekte der Behandlung von ZNS-Infektionen [35, 45]}

Antibiotika verteilen sich nicht gleichmäßig in den einzelnen Kompartimenten des zentralen Nervensystems (Liquor, Extrazellulärraum des Hirngewebes, Intrazellularraum von Neuronen und Stützzellen). Außerdem kann man innerhalb des Liquorraumes einen ventrikulolumbalen Gradienten mit lumbal erhöhten Albumin- und Medikamentenkonzentrationen nachweisen. Die wichtigsten Parameter zur Bestimmung der Penetration in den Liquorraum sind die Liquor/Serum-Quotienten für die Konzentrationen im „steady state“ und für die AUC („Area under the cur- ve“). Häufig ist die Halbwertzeit des Liquorraumes länger als die im Serum gemessene, so dass bei der Medikation hohe Dosen möglicherweise gegenüber kurzen Dosierungsabständen eine größere Rolle spielen. Für die Penetration des Liquor und des Hirngewebe sind für die meisten Antibiotika die Lipophilie, das molekulare Gewicht und die Serumeiweißbindung entscheidend. Bei intakter Blut-Liquor und Blut-Hirn-Schranke ist die Penetration hydrophiler Antibiotika wie z. B. $\beta$-Laktam-Antibiotika und Glykopeptide deutlich vermindert und steigt während einer meningealen Entzündung an. Im Gegensatz dazu sind lipophile Substanzen (Metronidazol, Chinolone, Rifampicin) weniger von der Schrankenfunktion abhängig. Die Bestimmung der minimalen inhibitorischen Konzentration eines Antibiotikums ist besonders wichtig, da für eine rasche Sterilisation 10-mal so hohe Wirkstoffkonzentrationen der minimalen inhibitorischen Konzentration notwendig sind. Wie groß der Quotient minimale Wirkstoffkonzentration/minimale inhibitorische Konzentration mindestens sein muss, ist noch unklar. Um optimale antibakterielle Konzentrationen trotz Veränderungen der Blut-Hirn- und Blut-Liquor-Schranke zu erreichen, wendet man vornehmlich nebenwirkungsarme Antibiotika, mäßig lipophile Substanzen mit niedriger Proteinbindung und vereinzelt die Kombination intravenöser und intraventrikulärer Darreichung an. Das Wichtigste Fazit für die antimikrobielle Therapie im ZNS sind:

- Aufgrund der primär verminderten Phagozytoseaktivität sollten in vitro bakterizide Medikamente angewendet werden

- Vermeiden einer Kombination von einem bakteriostatischen mit einem bakteriziden Medikament, da die bakteriostatisch

Tab. 5 Empfohlene Dosierung bei ZNS-Infektionen [2, 35, 44, 47, 56]

\begin{tabular}{|c|c|c|c|c|c|}
\hline $\begin{array}{l}\text { antibiotische } \\
\text { Substanz }\end{array}$ & $\begin{array}{l}\text { Tagesdosis } \\
\text { pro kg KG }\end{array}$ & $\begin{array}{l}\text { Dosierungs- } \\
\text { intervall }\end{array}$ & $\begin{array}{l}\text { Tagesdosis } \\
\text { Erwachsene }\end{array}$ & $\begin{array}{l}\text { AUC - Liquor-Serum- } \\
\text { quotient }\end{array}$ & $\begin{array}{l}\text { maximaler Anteil der } \\
\text { CCSF an der CSerum (\%) }\end{array}$ \\
\hline Ampicillin & $300-400 \mathrm{mg}$ & $\mathrm{q} 8 \mathrm{~h}$ & $12-15 \mathrm{~g}$ & & 14 \\
\hline Azlocillin & $300 \mathrm{mg}$ & $\mathrm{q} 8 \mathrm{~h}$ & و & & \\
\hline Cefepim & $150 \mathrm{mg}$ & q $12 \mathrm{~h}$ & $6 \mathrm{~g}$ & & 58 \\
\hline Cefotaxim & $200 \mathrm{mg}$ & $\mathrm{q} 8 \mathrm{~h}$ & $8-12 \mathrm{~g}$ & 0,12 & \\
\hline Ceftriaxon & $80-100 \mathrm{mg}$ & q $12 \mathrm{~h}$ & $4 \mathrm{~g}$ & 0,009 & 9 \\
\hline Ceftazidim & $150-200 \mathrm{mg}$ & $\mathrm{q} 8 \mathrm{~h}$ & $6-8 g$ & 0,057 & \\
\hline Chloramphenicol & $50-80-100 \mathrm{mg}$ & $\mathrm{q} 6 \mathrm{~h}$ & $50-100 \mathrm{mg} / \mathrm{kg}$ & & \\
\hline Ciprofloxacin & $20 \mathrm{mg}$ & $\mathrm{q} 8 \mathrm{~h}$ & $1,2 \mathrm{~g}$ & 0,26 & 26 \\
\hline Flucloxacillin & $200-300 \mathrm{mg}$ & $\mathrm{q} 8 \mathrm{~h}$ & $10-20 \mathrm{~g}$ & & \\
\hline Fosfomycin & $200 \mathrm{mg}$ & $\mathrm{q} 8 \mathrm{~h}$ & $10-15 \mathrm{~g}$ & 0,09 & \\
\hline Gentamicin & $5-6 \mathrm{mg}$ & q $24 \mathrm{~h}$ & و & & $0-30$ \\
\hline Meropenem & $150-200 \mathrm{mg}$ & $\mathrm{q} 8 \mathrm{~h}$ & $6 \mathrm{~g}$ & 0,047 & 21 \\
\hline Mezlocillin & $300 \mathrm{mg}$ & $\mathrm{q} 8 \mathrm{~h}$ & $10-20 \mathrm{~g}$ & & \\
\hline Metronidazol & $30 \mathrm{mg}$ & q $12 \mathrm{~h}$ & $1,2 \mathrm{~g}$ & & \\
\hline Netilmicin & $7,5 \mathrm{mg}$ & q $24 \mathrm{~h}$ & $0,6 \mathrm{~g}$ & & 26 \\
\hline Penicillin G & $250-400-500 \mathrm{kE}$ & $\mathrm{q} 6 \mathrm{~h}$ & 10-20 Mio E & & 10 \\
\hline Piperacillin & $300 \mathrm{mg}$ & $\mathrm{q} 8 \mathrm{~h}$ & g & 0,051 & \\
\hline Rifampicin & $20 \mathrm{mg}$ & $\mathrm{q} 8 \mathrm{~h}$ & $750 \mathrm{mg}$ & 0,25 & 56 \\
\hline TMP-SMZ & $20 \mathrm{mg}$ & $q 6 h$ & $20 \mathrm{mg} / \mathrm{kg} \mathrm{KG}$ & 0,15 & \\
\hline Vancomycin & $60 \mathrm{mg}$ & $\mathrm{q} 6 \mathrm{~h}$ & $2-3 g$ & & 14 \\
\hline
\end{tabular}


wirksame Substanz die Bakterizidie der anderen beeinträchtigen kann

- Wenn eine intrathekale Applikation notwendig ist, sollte dies aufgrund des ventrikulolumbalen Gradienten über ein intraventrikuläres System erfolgen

- bei Hinweisen auf eine zerebrale Manifestation einer Infektion, Anwendung der adäquaten ZNS-Dosis, d.h. in der Regel die höchstmögliche Dosis (Tab. 5).

\section{Intrathekale antimikrobielle Therapie}

In Ausnahmefällen wird bei zerebralen Infektionen auch die intrathekale Applikation von Antibiotika, meist unter Benutzung eines Ommaya-Reservoirs, empfohlen. Erfahrungen gibt es hierzu bei Infektionen mit Methicillin-resistenten und sensiblen Staphylokokken [12, 41]. Kommt es während der systemischen Therapie mit Vancomycin nicht zur Entfieberung, ist der klinische und/oder bildgebende Verlauf progredient, bzw. ist der Liquor in Kontrollpunktionen weiterhin positiv, kann bei bekannter schlechter Penetration der Blut-Hirn-Schranke durch Vancomycin die direkte intrathekale Gabe erfolgen. Die Empfehlungen zu Dosis, Dauer und Liquorspiegeln sind kontrovers. Die mehrheitlich empfohlene Dosis beträgt einmal täglich $10 \mathrm{mg}$ für Säuglinge und $20 \mathrm{mg}$ für Schulkinder [41, 50]. Die Dauer der Behandlung beträgt 7 Tage. Die Liquorkultur sollte im Regelfall schon nach 2-3 Gaben steril sein. Die im Liquor gemesssenen Talspiegel sollten bei $5 \mu \mathrm{g} / \mathrm{ml}$ liegen [3]. Aufgrund der nachgewiesenen ototoxischen Potenz von intrathekal appliziertem Vancomycin müssen Hörtests erfolgen [29]. Da bei alleiniger intrathekaler Vancomycingabe die entsprechenden Spiegel im Serum extrem niedrig sind, empfehlen einige Autoren parallel zur intrathekalen auch die systemische Gabe von Vancomycin, um die Entwicklung von Resistenzen zu vermeiden [50]. Für die intrathekale Anwendung von Imipenem als auch Penicillin besteht eine relative Kontraindikation aufgrund seiner epileptogenen Potenz bei Kindern mit bakterieller Meningitis. Dies gilt nicht für Meropenem [21].

Einige wenige Fallbeschreibungen gibt es zur intrathekalen Behandlung mit Quinpristin/Dalfopristin bei Shuntinfektionen mit Vancomycin-resistentem E. faecium über den Shunt [67, 72]. Die Indikation entsprach der von i.th. Vancomycin, d. h. wurde aufgrund der bekannten schlechten Penetration durch die Bluthirnschranke mit der alleinigen i.v. Gabe von Quinpristin/Dalfopristin keine Liquorsanierung erreicht, wurde die systemische um die intrathekale Therapie mit 1-5 mg täglich für 3-8 Tage erweitert. Allerdings kam es zum Teil zu erheblichen toxischen Enzephalopathien mit Hirnödem, Hydrozephalus und zerebralen Infarkten [26]. Daten zu Kindern gibt es für diese Anwendung bisher nicht. Im Weiteren werden auch intrathekale Gaben über Reservoire mit intraventrikulären Schenkeln von Gentamicin, Methicillin [41], Arbekacin bei Methicillin-resistentem Staphylococcus aureus [39], Polymixin B bei Ceftazidim-resistenten Klebsiellen [58] und Colistin bei Carbapenem-resistentem Acinetobacter [22] berichtet. Dies sind ausnahmslos Kasuistiken. Es gibt bisher nur eine randomisierte Studie, die die Wertigkeit einer intraventrikulären antibiotischen Therapie im pädiatrischen Bereich evaluiert. Hierbei ergab sich, dass intraventrikuläre antibiotische Therapie zusätzlich zur intravenösen antibiotischen Therapie bei gramnegativer Meningitis pädiatrischer immunkompetenter Patienten sogar mit einer erhöhten Mortalität im
Vergleich zur Kontrollgruppe mit alleiniger intravenöser Behandlung assoziiert war. Infolgedessen wurde in dieser Cochrane-Analyse von einer intraventrikulären antibiotischen Therapie abgeraten [60].

Auch Amphotericin B penetriert die Blut-Hirn-Schranke schlecht. Gelingt die antimykotische Sanierung nicht mit der systemischen Therapie, gibt es einzelne Berichte, diese durch die intrathekale Applikation von Amphotericin B zu erzielen [9, 11, 20, 31]. Die Anwendung wurde im Regelfall mit Hydrokortison kombiniert. Daten zur Höchstdosis, Clearance oder Therapiedauer gibt es bisher nicht. Als Nebenwirkung der i.th. Amphotericin-B-Therapie sind Fieber, Schüttelfrost, Kopfschmerz, Übelkeit, Erbrechen, Glieder- und Muskelschmerzen sowie Gewichtsverlust beschrieben. Liposomales Amphotericin B wurde bisher zur i.th. Therapie nicht eingesetzt. Im Tierversuch erweist es sich aber als deutlich weniger toxisch im Vergleich zum konventionellen Amphotericin B [14]. Eine Zulassung von Amphotericin $B$ für die i.th. Anwendung gibt es bisher nicht.

\section{Empirische Therapie einer ZNS-Infektion}

Die Behandlung klinisch und/oder mikrobiologisch ungesicherter zerebraler Infektionen erfolgt nach validierten Therapiekonzepten wie sie auch für die Therapie unklaren Fiebers gelten [32, $37,56,57]$. Zerebrale Infektionen sind aber insgesamt eine seltene Ursache unklaren Fiebers in der Granulozytopenie (<1\%) [62]. Das Erregerspektrum ist dem bei unklaren Fieber in der Granulozytopenie gleich. Die Lumbalpunktion zur Liquordiagnostik sollte unbedingt vor Beginn der empirischen antimikrobiellen Therapie erfolgen, da sonst die Rate positiver Befunde deutlich abnimmt. Ein sofortiger Behandlungsbeginn ist prognostisch entscheidend [66]. Bei Patienten mit fokalen neurologischen Ausfällen bzw. Papillenödem sollte vor einer Lumbalpunktion möglichst eine bildgebende Untersuchung erfolgen und vorher die empirische Therapie begonnen werden, da bei Verzögerung die Mortalität nachweislich ansteigt $[5,68]$. Weitere Kontraindikationen für eine Lumbalpunktion sind bildgebende Hinweise auf erhöhten intrakraniellen Druck und klinische Zeichen der Herniation.

Aufgrund des Erregerspektrums bei Hirnabszessen sollte immer bis zur Klärung des Erregers eine antibakterielle mit einer antimykotischen Therapie verbunden werden. In den letzten Jahren hat sich antibakteriell die Kombination aus Cefotaxim/Cetriaxon mit Metronidazol als sinnvoll erwiesen. Auch die zerebrale Toxoplasmose besitzt eine Neigung zur Abszessbildung, spielt aber, im Gegensatz zu Patienten mit AIDS, bei hämatologisch/onkologischen Patienten nur eine untergeordnete Rolle. Die Therapie besteht in einer Kombination aus Sulfadiazin und Pyrimethamin [19]. Weitere Therapieoptionen sind im Kapitel zu Parasitosen aufgeführt. Zerebritische Stadien können noch alleine antimikrobiell behandelt werden, im weiteren Verlauf ist jedoch die chirurgische Intervention notwendig, da ansonsten die Mortalität und Morbidität sprunghaft ansteigt [54].

\section{Erregerspezifische Therapie von Bakterien, Pilzen und Viren} Hierzu gibt es ausführliche Empfehlungen im Kapitel der Behandlung einzelner Erreger. 


\section{Empirische Therapie der Infektion von intrazerebralen Katheterinfektionen}

Bei Patienten mit intrazerebralen Kathetern (Rickham-Reservoir, Ventrikel-Shunt, Ommaya-Reservoir) muss neben Staphylococcus aureus und Koagulase-negativen Staphylokokken (KNS) (meist Staphylococcus epidermidis) auch mit einer Infektion durch gramnegative Erreger (einschließlich Pseudomonas) gerechnet werden [40]. Für KNS besteht eine Wirksamkeitslücke in der empirischen initialen Antibiotika-Therapie, so dass die zusätzliche Gabe eines Glykopeptids notwendig wird, eventuell kombiniert mit dem gut liquorgängigen Rifampicin [1]. Selten ist die Shuntinfektion durch anaerobe Keime. Dabei handelt es sich einerseits um Keime der Hautflora (Propionibacterium acnes), andererseits um Keime des Gastrointestinaltraktes (Lactobacillus), die bei Perforationen des Darmes durch den peritonealen Anteil des Shuntes aszendieren [6]. Bei Resistenz gegenüber Penicillinen sind die gut liquorgängigen Antibiotika Metronidazol, Chloramphenicol und Meropenem wirksam [7]. Manche Autoren kommen zu dem Schluss, dass eine alleinige antibiotische Therapie zur nachhaltigen Keim-Sanierung häufig ausreicht $[12,41]$. Anderen Autoren reicht bei einer Shuntinfektion in der Regel die alleinige antibiotische Therapie nicht aus. In 50-80\% der Fälle ließ sich die Fremdkörperinfektion nur durch die zusätzliche Shunt-Explantation und vorübergehende Anlage einer externen Liquordrainage nachhaltig sanieren [74]. Etwas geringer waren die Angaben in einer Untersuchung bei einem pädiatrisch-onkologischen Kollektiv mit Meningitis bei liegendem ZNS-Ableitungssystem: nur in 25\% der Fälle war eine Keimsanierung erst nach Explantation des Shuntsystem möglich [62]. Zusammengefasst liegen keine ausreichend kontrollierten Daten vor, so dass die Empfehlung, die Sanierung des Liquors nach Entfernung des Shuntsystems zu erreichen und hernach eine Reimplantation erwägen, lauten muss.

\section{Unerwünschte Wirkungen der antimikrobiellen Therapie auf das ZNS}

Das Ansprechen der Erreger auf die Therapie wird vordergründig anhand der Funktionsprüfung des ZNS beurteilt. Dabei muss man beachten, dass antimikrobielle Substanzen folgende Komplikationen auslösen können [53]:

- Epileptische Anfälle (Penicilline, Amphothericin B, Metronidazol, Ciprofloxacin, Acyclovir, Foscarnet, Praziquantel, Isoniazid)

- Bewusstseinstrübung (Ciprofloxacin, Ofloxacin, Rifampicin, Flucytosin, Acyclovir, Foscarnet, Isoniazid)

- Hörstörung (Aminoglykoside, Erythromycin, Azithromycin, Minocyclin)

- Periphere Neuropathie ( Metronidazol, Amphothericin B, Isoniazid)

\section{Supportivmaßnahmen}

\section{Antiinflammatorische Therapie}

In klinischen Studien bei Kindern mit H.-influenzae-b- und Pneumokokken-Meningitis jenseits der 8. Lebenswoche konnte außerhalb des onkologischen Krankenguts nachgewiesen werden, dass der Einsatz von Dexamethason den intrakraniellen Druck, die überschießende Zytokin-vermittelte Entzündungsreaktion, sowie das Hirnödem reduziert. Damit verbunden ist eine signifi- kante Reduktion der audiologischen und neurologischen Spätschäden [69, 71]. Bei der Meningokokken-Meningitis sind die Daten unzureichend. Eine Dexamethasontherapie kann über 2 bzw. 4 Tage mit je 2-mal 0,4 bzw. 0,2 mg/kg KG erwogen werden [65]. Die erste Gabe des Glukokortikoids sollte 15-20 min vor oder zumindest gleichzeitig mit der ersten Antibiotika-Gabe erfolgen. Auch bei der Tbc-Meningitis gibt es Hinweise, dass die Behandlung mit Kortikosteroiden den neurologischen Endstatus verbessert [17]. Im Erwachsenenbereich ist lediglich bei bakterieller Meningitis und Liquorzellzahl > 1000/ $\mu$ l eine Dexamethasonprophylaxe ausreichend gesichert [47].

Die Gabe von Kortikosteroiden beim Hirnabszess wird kontrovers diskutiert. Bei Nachweis eines deutlich raumfordernden perifokalen Ödems wird von vielen Autoren zur Senkung des intrakraniellen Drucks Dexamethason empfohlen. Die Dauer variiert zwischen wenigen Tagen und 2 Wochen [64]. Neue Untersuchungen am Tiermodell zeigen allerdings, dass Dexamethason bei der Meningitis die neuronale Apoptose im Hippocampus verstärken kann [44]. Werden in dieser Richtung weitere Erkenntnisse gewonnen, muss in Zukunft möglicherweise erneut eine Risiko-Nutzen-Abwägung hinsichtlich einer Kortikosteroidtherapie erfolgen. Bei der zerebralen Toxoplasmose wird bis zum Abklingen der akuten Symptome die Einnahme von Glukokortikoiden empfohlen [19]. Diese Daten wurden an immunkompetenten Patienten gewonnen. Ob sie auf das pädiatrisch-onkologische Patientengut übertragen werden kann, ist nicht untersucht. Eine Kortikoidsteroidtherapie kann bei beginnender Regeneration der Knochenmarkfunktion eine mögliche positive Wirkung haben. Wichtig ist zu bedenken, dass bei Gabe von Kortikosteroiden die Liquorgängigkeit von Glykopeptiden vermindert ist und entsprechend andere Antibiotikakombinationen empfehlenswert sind [49].

\section{Antikonvulsive Therapie}

Zerebrale Anfälle können in bis zu $40 \%$ bei pädiatrischen immunkompetenten Patienten im Rahmen einer bakteriellen Meningitis auftreten. Diese führen zu einer Erhöhung des intrakraniellen Druckes und verbrauchen die zerebralen Energiereserven. Eine vermehrte Anfallsaktivität führt somit zur Aggravation von zytotoxischer als auch vasogener Ödembildung. Daher empfiehlt es sich, die Patienten rechtzeitig antikonvulsiv mit Lorazepam, Diazepam, Phenobarbital oder Phenytoin zu behandeln [2, 52]. Anfälle waren in einer Serie von pädiatrisch-onkologischen Patienten mit ZNS-Infektionen signifikant mit erhöhter Mortalität und Morbidität assoziiert [62].

\section{Chirurgische Maßnahmen}

Die definitive Diagnose eines Abszesses erfordert eine stereotaktische Punktion mit mikroskopischer und kultureller Untersuchung [53]. Die Feinnadelaspiration ist bei normalen Gerinnungsverhältnissen ein Eingriff mit nur geringer Komplikationsrate mit einer Morbidität bzw. Mortalität von jeweils 0,7\% [24]. Eine primär antibiotische und antimykotische Therapie kommt beim zerebritischen Frühstadium vor Bildung der Abszessmembran, bei inoperablen Patienten in schlechtem Allgemeinzustand, bei Hirnstammabszessen, bei Abszessen unter $2(-3) \mathrm{cm}$, oder auch bei Patienten mit multiplen Abszessen in Betracht. 
Hier bleibt nach stereotaktischer Aspiration des größten Herdes zu Diagnosezwecken meist nur die alleinige antibiotische Therapie [63]. Für alle übrigen Fälle wird für die Therapie des Hirnabszess generell ein kombiniertes therapeutisches Vorgehen empfohlen, d.h. antimikrobielle Therapie und Chirurgie [38]. Die weltweit am meisten akzeptierten Richtlinien sehen eine Indikation zur Aspiration oder Exzision ab einer Abszessgröße von $2,5 \mathrm{~cm}$ im Durchmesser [10,36]. Handelt es sich um eine rhinozerebrale Mukormykose, sollte zudem, wo anatomisch möglich (Nasenhöhle, Nasennebenhöhle, Knorpel), ein Débridement erfolgen [46].

Bei ca. ${ }^{1 / 3}$ der Patienten mit Meningitis ist ein subduraler Erguss nachweisbar. Dieser ist meist symptomlos und spontan regredient. Eine Indikation für eine Nadelpunktion besteht bei klinischem Verdacht eines Empyems, einem schnell zunehmenden Kopfumfang ohne Hydrozephalus, fokalen neurologischen Symptomen und Hinweisen auf erhöhten Hirndruck.

\section{Monitoring}

Die Meningitis/Enzephalitis erfordert bereits bei Verdacht eine stationäre und gegebenenfalls intensivmedizinische Überwachung. Diese kann das Monitoring des Hirndrucks, evtl. mit einer Hirndrucksonde, das Monitoring von Elektrolyten und Ausscheidung zur Erfassung einer inadäquaten ADH-Produktion, sowie das Monitoring von neurologischem Status und Krampfbereitschaft ermöglichen.

Bei mikrobiologisch und/oder klinisch gesicherten Infektionen sollte zur Sicherung des Behandlungserfolges noch während der antimikrobiellen Therapie eine Wiederholung der Diagnostik erfolgen (Liquor, CT, MRT). Bei einer unkomplizierten Meningitis mit rascher klinischer Besserung nach Beginn der antibiotischen Therapie verzichten einige Autoren auf eine Kontrollpunktion des Liquors. Die Mehrheit empfiehlt aber zur Therapiekontrolle eine erneute Liquorentnahme nach $48 \mathrm{~h}$. In jedem Falle sollte eine Wiederholung der Punktion bei Auftreten neuer neurologischer Symptome stattfinden [15]. Sie sollte insbesondere auch dann erwogen werden, wenn gleichzeitig die Gabe von Dexamethason erfolgt, da Kortikosteroide die klassischen Parameter des Behandlungserfolges (Fieber, Entzündungszeichen, Ödem) verschleiern [49]. Insbesondere können durch hohe Dosen Dexamethason auch zuvor erfolgreich behandelte Erkrankungen reaktiviert werden. Bei entsprechender Anamnese sind sogar serielle Lumbalpunktionen gerechtfertigt [53]. Da bei sehr kleinen Kindern die klinische und anamnestische Exploration nur unzureichende Daten liefert, empfehlen manche Autoren nach Absetzen der Antibiotika eine nochmalige Liquorpunktion zur Kontrolle des Therapieerfolges durchzuführen, eventuell kombiniert mit einer bildgebenden Untersuchung, um intrakranielle Komplikationen auszuschließen [55]. Gerade die bildgebenden Untersuchungen (MRT/CT) haben ihren Stellenwert weniger bei der primären Diagnostik der Meningitis/Enzephalitis, sondern vielmehr in deren Verlaufskontrolle. Komplikationen wie Hydrozephalus, Hirninfarkt, Sinusvenenthrombose und Hirnabszess können hierdurch erfasst und verlaufsdokumentiert werden. Insbesondere das MRT eignet sich sehr gut zur Stadiendokumentation eines Hirnabszesses. Um die Regredienz des Abszesses zu verfolgen werden MRT- bzw. CT-Kontrollen in ca. 2-wöchigen Abständen empfohlen [27, 53].

Zu den Basismaßnahmen der Hirnödem-Therapie gehören das „minimal handling“, Kopfmittellage bei $30^{\circ}$ hochgelagertem Oberkörper, eine suffiziente Antipyrese, Normovolämie, Normotonie, Normonatriämie und eine Analgosedierung. Die Entscheidung zur Intubation und Beatmung sollte frühzeitig gestellt werden, angestrebt ist die Normoventilation. Die Hyperventilation zur Senkung des intrakraniellen Blutflusses ist bei Kindern obsolet, da es mittelfristig zum Rebound des Hirnödems kommt [55]. Die intrakranielle Druckmessung kann intraparenchymatös per Camino-Druck-Sonde oder mit einem intraventrikulär platzierten Katheter erfolgen. Spezielle Maßnahmen zur ICP-Senkung sind Diuretika kombiniert mit Mannitol 20\%, in Einzelfällen auch die Gabe von Natrium, eine milde Hyperventilation und die supranarkotische Barbiturattherapie.

Grundsätzlich muss die Ätiologie des intrakraniellen Druckes geklärt werden, um kausale Maßnahmen einzuleiten. Der Hydrocephalus communicans ist eine häufige Komplikation. Die frühzeitige Anlage einer Drainage zur Entlastung von Liquor trägt wesentlich zur Senkung der Mortalität und der neurologischen Spätschäden bei.

\section{Akute Komplikationen}

Die Mortalität der bakteriellen ZNS-Infektion beim immunkompetenten Kind liegt um 5-10\% [33]. Beim erwachsenen onkologischen Patienten liegt sie deutlich höher mit 60\% [53]. Insbesondere bei Pilzabszessen liegt sie mit 42-77\% extrem hoch [23]. Bei der zerebralen Aspergillose liegt sie sogar bei über 90\% [16]. Bei onkologischen Patienten aus der pädiatrischen Gruppe gibt es bisher nur eine monozentrische retrospektive Studie, die sich mit den späten Komplikationen und der Mortalität der bakteriellen und fungalen Meningitis befasst. Bei den 40 untersuchten Kindern lag die Mortalität bei $13 \%$. Die Neutropenie als Risikofaktor spielt eine wesentliche Rolle: Alle fünf Patienten, die an den Folgen der Meningitis verstarben, stammten aus dieser Gruppe (entsprechend 48\%) [62]. Die Angaben zur Meningitisbedingten Mortalität bei erwachsenen Patienten mit Neutropenie liegen mit 86\% deutlich höher [34].

Kreislaufprobleme entwickeln sich meist während der frühen Phase der Meningitis. Der Schock ist eine ernste Komplikation der Meningitis [28]. In der Vergangenheit wurde angenommen, dass es bei Patienten mit Meningitis bzw. Enzephalitis zu einer vermehrten $\mathrm{ADH}$-Ausschüttung (Syndrom der inadäquaten ADH-Sekretion) kommt. Die Folge ist eine hypotone Hyperhydratation mit akuter Hyponatriämie. In experimentellen und klinischen Untersuchungen zeigte sich allerdings, dass die erhöhten ADH-Werte eher auf eine adäquate Hormonantwort auf nicht registrierte Hypovolämien hindeuten, und dass dementsprechend in dieser Situation eher großzügige Volumensubstitutionen therapeutisch indiziert sind [43]. Diese Erkenntnis ist wichtig, da der systemische Blutdruck auf einem Niveau gehalten werden sollte, der ausreicht, um eine ausreichende zerebrale Perfusion zu gewährleisten [18]. 
Ein Drittel der Patienten entwickelt vor, während und innerhalb der ersten Tage nach stationärer Aufnahme zerebrale Krampfanfälle. Obwohl die meisten Krampfanfälle generalisiert sind, sind fokale Krampfanfälle prognostisch in Bezug auf Mortalität und neurologische Spätschäden ungünstiger. Ebenfalls prognostisch ungünstig sind therapierefraktäre Anfälle, solche, die über den 4. Tag des stationären Aufenthalts hinaus noch persistieren, und solche, die erstmals zu einem späten Zeitpunkt auftreten $[28,62]$. Fokale neurologische Symptome wie Hemiparese, Tetraparese, Gesichtsnervenlähmungen und Sehstörungen können bereits in der frühen Krankheitsphase auftreten. Sie können durch einen kortikalen Nekrosebezirk, eine okklusive Vaskulitis oder eine Thrombose der kortikalen Venen verursacht sein. Nicht selten persistieren diese fokalen neurologischen Schäden [59]. Eine Ausbreitung des meningealen inflammatorischen Prozesses bis in den Subarachnoidalraum kann den II., III., VI.VIII. Hirnnerven miteinbeziehen [8].

Hirndruck als frühe Komplikation einer ZNS-Infektion kann sich rasch entwickeln und durch ein generalisiertes Hirnödem, eine Liquor-Abflussstörung (Blockierung der basalen Zisternen, entzündliche Adhäsion der Leptomeningen), eine venöse Abflussbehinderung (Sinusvenenthrombose), oder eine intrakranielle Raumforderung (Abszess) verursacht werden [73]. Gefürchtet, aber selten ist die Einklemmung der Tonsillae cerebelli. Die intrakranielle Hirndruckmessung in der Akutphase verbessert den neurologischen Endstatus wesentlich.

Pilze führen aufgrund eines hohen Angiotropismus zu typischen Komplikationen [13]. Bei Invasion mittlerer und großer Arterien und Venen kommt es durch Ausbildung breiter Hyphen zu Okklusionen mit Thrombosen oder zum Teil zu ausgedehnten nekrotisch-hämorrhagischen Infarkten und sekundären Abszessen [4, 46]. Mittelgroße Pseudomyzelien (Candida) okkludieren eher kleine Gefäße und führen durch Störung der Mikrozirkulation zu Nekrosen des umgebenden Gewebes und zu Mikroabszessen. Bisher gibt es keine gesicherte Behandlungsoption der septischen Thrombosen, die antikoagulative Therapie wird kontrovers diskutiert.

Hämorrhagische Hirninfarkte sind eine seltene Komplikation. Ursächlich ist die Produktion eines dickflüssigen Exsudates, das zu einer Vaskulitis mit Perforation der Gefäße und schließlich zum Hirninfarkt führen kann. Betroffen sind v.a. die Gefäße der Hirnbasis, insbesondere der Basalganglien.

In mehr als $1 / 3$ der Patienten mit Meningitis sind subdurale Ergüsse nachweisbar. Sie sind im Allgemeinen symptomlos, werden im Regelfall wieder resorbiert und hinterlassen gewöhnlich keine neurologische Schäden [61]. Nur sehr selten entwickelt sich ein Empyem.

\section{Literatur}

${ }^{1}$ Archer GL, Climo MW. Antimicrobial susceptibility of coagulase-negative staphylococci. Antimicrob Agents Chemother 1994; 38: 2231 2237

${ }^{2}$ Ashwal S. Neurologic evaluation of the patient with acute bacterial meningitis. Neurol Clin 1995; 13: 549-577
${ }^{3}$ Bayston R. Epidemiology, diagnosis, treatment, and prevention of cerebrospinal fluid shunt infections. Neurosurg Clin N Am 2001; 12: $703-708$

${ }^{4}$ Beal MF, O'Carroll CP, Kleinman GM et al. Aspergillosis of the nervous system. Neurology 1982; 32: $473-479$

${ }^{5}$ Bonsu BK, Harper MB. Fever interval before diagnosis, prior antibiotic treatment, and clinical outcome for young children with bacterial meningitis. Clin Infect Dis 2001; 32: 566-572

${ }^{6}$ Brook I. Anaerobic infections in children. Microbes Infect 2002; 4: $1271-1280$

${ }^{7}$ Brook I. Meningitis and shunt infection caused by anaerobic bacteria in children. Pediatr Neurol 2002; 26: 99-105

${ }^{8}$ Brookhouser PE, Auslander MC, Meskan ME. The pattern and stability of postmeningitic hearing loss in children. Laryngoscope 1988; 98: $940-948$

${ }^{9}$ Buxhofer V, Ruckser R, Kier P et al. Successful treatment of invasive mould infection affecting lung and brain in an adult suffering from acute leukaemia. Eur J Haematol 2001; 67: 128-132

${ }^{10}$ Calfee DP, Wispelwey B. Brain abscess. Semin Neurol 2000; 20 : $353-360$

${ }^{11}$ Camarata PJ, Dunn DL, Farney AC et al. Continual intracavitary administration of amphotericin B as an adjunct in the treatment of aspergillus brain abscess: case report and review of the literature. Neurosurgery 1992; $31: 575-579$

12 Chamberlain MC, Kormanik PA, Barba D. Complications associated with intraventricular chemotherapy in patients with leptomeningeal metastases. J Neurosurg 1997; 87: 694-699

${ }^{13}$ Chimelli L, Mahler-Araujo MB. Fungal infections. Brain Pathol 1997; 7 : $613-627$

${ }^{14}$ Clemons KV, Sobel RA, Williams PL et al. Comparative toxicities and pharmacokinetics of intrathecal lipid (amphotericin B colloidal dispersion) and conventional deoxycholate formulations of amphotericin B in rabbits. Antimicrob Agents Chemother 2001; 45: 612-615

15 de Montalembert M. [Bacterial meningitis in children: how many lumbar punctures?]. Pediatrie 1993; 48: $17-19$

${ }^{16}$ Denning DW, Stevens DA. Antifungal and surgical treatment of invasive aspergillosis: review of 2121 published cases. Rev Infect Dis 1990; 12 : $1147-1201$

17 Dooley DP, Carpenter JL, Rademacher S. Adjunctive corticosteroid therapy for tuberculosis: a critical reappraisal of the literature. Clin Infect Dis 1997; 25: 872-887

${ }^{18}$ Duke T, Mokela D, Frank D et al. Management of meningitis in children with oral fluid restriction or intravenous fluid at maintenance volumes: a randomised trial. Ann Trop Paediatr 2002; 22: 145-157

${ }^{19}$ Einsele H, Bertz H, Beyer J et al. Infectious complications after allogeneic stem cell transplantation: epidemiology and interventional therapy strategies - guidelines of the Infectious Diseases Working Party (AGIHO) of the German Society of Hematology and Oncology (DGHO). Ann Hematol 2003; 82: S175-S 185

20 Elgamal EA, Murshid WR. Intracavitary administration of amphotericin B in the treatment of cerebral aspergillosis in a non immune-compromised patient: case report and review of the literature. Br J Neurosurg 2000; 14: 137-141

${ }^{21}$ Farr BM, Scheld WM. Nosocomial meningitis. Serious Hospital Inf 1998; $10: 1-7$

${ }^{22}$ Fernandez-Viladrich P, Corbella X, Corral L et al. Successful treatment of ventriculitis due to carbapenem-resistant Acinetobacter baumannii with intraventricular colistin sulfomethate sodium. Clin Infect Dis 1999; 28: $916-917$

${ }^{23}$ Guppy KH, Thomas C, Thomas K et al. Cerebral fungal infections in the immunocompromised host: a literature review and a new pathogen Chaetomium atrobrunneum: case report. Neurosurgery 1998; 43: $1463-1469$

${ }^{24}$ Hall WA, Martinez AJ, Dummer JS et al. Nocardial brain abscess: diagnostic and therapeutic use of stereotactic aspiration. Surg Neurol 1987; 28 : $114-118$

${ }^{25}$ Hedlund GL, Boyer RS. Neuroimaging of postnatal pediatric central nervous system infections. Semin Pediatr Neurol 1999; 6: 299-317

${ }^{26}$ Kanchanapoom T, Koirala J, Goodrich J et al. Treatment of central nervous system infection by vancomycin-resistant enterococcus faecium. Diagn Microbiol Infect Dis 2003; 45: 213-215

27 Kaplan K. Brain abscess. Med Clin North Am 1985; 69: 345-360

${ }^{28}$ Kaplan SL. Clinical presentations, diagnosis, and prognostic factors of bacterial meningitis. Infect Dis Clin North Am 1999; 13: 579- 594 
${ }^{29}$ Klibanov OM, Filicko JE, DeSimone Jr JA et al. Sensorineural hearing loss associated with intrathecal vancomycin. Ann Pharmacother 2003; 37: $61-65$

${ }^{30}$ Krupova Y, Sejnova D, Dzatkova J et al. Prospective study on fungemia in children with cancer: analysis of 35 cases and comparison with 130 fungemias in adults. Support Care Cancer 2000; 8: 427-430

${ }^{31}$ LePage E. Using a ventricular reservoir to instill amphotericin B. J Neurosci Nurs 1993; 25: $212-217$

${ }^{32}$ Link H, Bohme A, Cornely OA et al. Antimicrobial therapy of unexplained fever in neutropenic patients - guidelines of the Infectious Diseases Working Party (AGIHO) of the German Society of Hematology and Oncology (DGHO), Study Group Interventional Therapy of Unexplained Fever, Arbeitsgemeinschaft Supportivmassnahmen in der Onkologie (ASO) of the Deutsche Krebsgesellschaft (DKG-German Cancer Society). Ann Hematol 2003; 82: S105-S117

${ }^{33}$ Lipton JD, Schafermeyer RW. Evolving concepts in pediatric bacterial meningitis - Part I: Pathophysiology and diagnosis. Ann Emerg Med 1993; 22: $1602-1615$

${ }^{34}$ Lukes SA, Posner JB, Nielsen S et al. Bacterial infections of the CNS in neutropenic patients. Neurology 1984; 34: 269-275

${ }^{35}$ Lutsar I, McCracken Jr GH, Friedland IR. Antibiotic pharmacodynamics in cerebrospinal fluid. Clin Infect Dis 1998; 27: 1117-1127

${ }^{36}$ Marchiori C, Tonon E, Boscolo Rizzo P et al. [Brain abscesses after extracranial infections of the head and neck area]. Hno 2003; 51: $813-$ 822

${ }^{37}$ Maschmeyer G. Interventional antimicrobial therapy in febrile neutropenic patients. Diagn Microbiol Infect Dis 1999; 34: 205-212

38 Mathisen GE, Johnson JP. Brain abscess. Clin Infect Dis 1997; 25 : $763-779$

${ }^{39}$ Matsubara H, Makimoto A, Higa T et al. Successful treatment of meningoencephalitis caused by methicillin-resistant Staphylococcus aureus with intrathecal vancomycin in an allogeneic peripheral blood stem cell transplant recipient. Bone Marrow Transplant 2003; 31 $65-67$

${ }^{40}$ Mayhall CG, Archer NH, Lamb VA et al. Ventriculostomy-related infections. A prospective epidemiologic study. N Engl J Med 1984; 310: $553-559$

${ }^{41}$ Mechleb B, Khater F, Eid A et al. Late onset Ommaya reservoir infection due to Staphylococcus aureus: case report and review of Ommaya Infections. J Infect 2003; 46: 196- 198

${ }^{42}$ Mikrobiologie DGfHu. Infektionen des Zentralnervensystems. Qualitätsstandards in der mikrobiologisch-infektiologischen Diagnostik. Urban und Fischer, München 2001

${ }^{43}$ Moller K, Larsen FS, Bie P et al. The syndrome of inappropriate secretion of antidiuretic hormone and fluid restriction in meningitis - how strong is the evidence? Scand J Infect Dis 2001; 33: 13-26

${ }^{44}$ Nau R, Bruck W. Neuronal injury in bacterial meningitis: mechanisms and implications for therapy. Trends Neurosci 2002; 25: 38-45

${ }^{45}$ Nau R, Sorgel F, Prange HW. Pharmacokinetic optimisation of the treatment of bacterial central nervous system infections. Clin Pharmacokinet 1998; 35: $223-246$

${ }^{46}$ Nenoff P, Kellermann S, Schober R et al. Rhinocerebral zygomycosis following bone marrow transplantation in chronic myelogenous leukaemia. Report of a case and review of the literature. Mycoses 1998; 41: $365-372$

${ }^{47}$ Neurologie DGf. Leitlinien für Diagnostik und Therapie in der Neurologie. 2. Überarbeitung 2003

${ }^{48}$ Neuropädiatrie Gf. Deutsche Gesellschaft für Kinderheilkunde und Jugendmedizin. Leitlinien Kinderheilkunde und Jugendmedizin. Urban und Fischer, München 2001

${ }^{49}$ Paris MM, Hickey SM, Uscher MI et al. Effect of dexamethasone on therapy of experimental penicillin- and cephalosporin-resistant pneumococcal meningitis. Antimicrob Agents Chemother 1994; 38: 1320 1324

50 Pfausler B, Spiss H, Beer R et al. Treatment of staphylococcal ventriculitis associated with external cerebrospinal fluid drains: a prospective randomized trial of intravenous compared with intraventricular vancomycin therapy. J Neurosurg 2003; 98: 1040-1044
${ }^{51}$ Pfisterer W, Muhlbauer M, Czech T et al. Early diagnosis of external ventricular drainage infection: results of a prospective study. J Neurol Neurosurg Psychiatry 2003; 74: 929-932

52 Pomeroy SL, Holmes SJ, Dodge PR et al. Seizures and other neurologic sequelae of bacterial meningitis in children. N Engl J Med 1990; 323: $1651-1657$

53 Pruitt AA. Nervous system infections in patients with cancer. Neurol Clin 2003; 21: $193-219$

54 Saez-Llorens X. Brain abscess in children. Semin Pediatr Infect Dis 2003; $14: 108-114$

55 Saez-Llorens X, McCracken Jr GH. Bacterial meningitis in children. Lancet 2003; 361: 2139-2148

56 Scholz H, Behloradsky BH, Heininger U et al. DGPI Handbuch Infektionen bei Kindern und Jugendlichen. 4. Aufl. Futuramed-Verlag, München 2004

${ }^{57}$ Schroten H. Diagnostik und Therapie der bakteriellen Meningitis. Monatsschrift Kinderheilkd 2004; 152: 382 - 390

${ }^{58}$ Segal-Maurer S, Mariano N, Qavi A et al. Successful treatment of ceftazidime-resistant Klebsiella pneumoniae ventriculitis with intravenous meropenem and intraventricular polymyxin $B$ : case report and review. Clin Infect Dis 1999; 28: 1134-1138

59 Sell SH. Long term sequelae of bacterial meningitis in children. Pediat Infect Dis 1983; 2: 90-93

${ }^{60}$ Shah S, Ohlsson A, Shah V. Intraventricular antibiotics for bacteria meningitis in neonates. Cochrane Database Syst Rev 2004; CD004496

61 Snedeker JD, Kaplan SL, Dodge PR et al. Subdural effusion and its relationship with neurologic sequelae of bacterial meningitis in infancy: a prospective study. Pediatrics 1990; 86: 163 - 170

62 Sommers LM, Hawkins DS. Meningitis in pediatric cancer patients: a review of forty cases from a single institution. Pediatr Infect Dis J 1999; 18: $902-907$

${ }^{63}$ Stapleton SR, Bell BA, Uttley D. Stereotactic aspiration of brain abscesses: is this the treatment of choice? Acta Neurochir (Wien) 1993; 121: $15-19$

64 Steiger HJ, Reuelen HJ. Manual Neurochirurgie. Ecomed, Landsberg 1999

65 Syrogiannopoulos GA, Lourida AN, Theodoridou MC et al. Dexamethasone therapy for bacterial meningitis in children: 2- versus 4-day regimen. J Infect Dis 1994; 169: 853 - 858

66 Talan DA, Zibulewsky J. Relationship of clinical presentation to time to antibiotics for the emergency department management of suspected bacterial meningitis. Ann Emerg Med 1993; 22: 1733-1738

67 Tan TY, Pitman I, Penrose-Stevens A et al. Treatment of a vancomycinresistant Enterococcus faecium ventricular drain infection with quinupristin/dalfopristin and review of the literature. J Infect 2000; 41: $95-97$

${ }^{68}$ Tunkel AR, Scheld WM. Acute bacterial meningitis. Lancet 1995; 346 : $1675-1680$

69 de Beek D van, de Gans J, McIntyre P et al. Corticosteroids in acute bacterial meningitis. Cochrane Database Syst Rev 2003; CD004305

70 den Berg H van, Gerritsen EJ, Haraldsson A et al. Changes in cell and protein content of cerebrospinal fluid in children with acute lymphoblastic leukaemia after allogeneic bone marrow transplantation. Bone Marrow Transplant 1993; 12: 615-619

${ }^{71}$ Wald ER, Kaplan SL, Mason Jr EO et al. Dexamethasone therapy for children with bacterial meningitis. Meningitis Study Group. Pediatrics 1995; $95: 21-28$

72 Williamson JC, Glazier SS, Peacock Jr JE. Successful treatment of ventriculostomy-related meningitis caused by vancomycin-resistant Enterococcus with intravenous and intraventricular quinupristin/dalfopristin. Clin Neurol Neurosurg 2002; 104: 54-56

${ }^{73}$ Yaramis A, Gurkan F, Elevli M et al. Central nervous system tuberculosis in children: a review of 214 cases. Pediatrics 1998; 102: E49

${ }^{74}$ Yogev R. Cerebrospinal fluid shunt infections: a personal view. Pediatr Infect Dis 1985; 4: 113-118 